\title{
'When Can I be Free from My Miserable Leg?' A Qualitative Study of Patients' Experiences of Chronic Leg Ulceration in Primary Healthcare
}

\author{
Xiaoli Zhu ${ }^{1 *}$ and Kath Ryan ${ }^{2}$ \\ ${ }^{1}$ Nursing Services, National Healthcare Group Polyclinics, Singapore \\ ${ }^{2}$ Social Pharmacy, University of Reading, United Kingdom \\ *Corresponding author: Xiaoli Zhu, Wound Care Nurse Clinician, Nursing Services, National Healthcare Group Polyclinics, \\ 3 Fusionopolis Link, Nexus@one-north (South Lobby), \#05-10, 138543, Singapore, Tel: (65)-63402447, Fax: (65)-6496- \\ 6753, E-mail: Julia_zhu@nhgp.com.sg
}

\begin{abstract}
Purpose: Chronic leg ulceration significantly reduces quality of life because of its chronicity and recurrence. This study aimed to gain deep insight into the experiences of patients living with chronic leg ulcer from their perspective.

Methods: A qualitative design including in-depth video or audio-recorded interviews, with eight participants in the primary healthcare setting was chosen to provide maximum variation of demographics and ulcer experience. Interviews, lasting up to 60 minutes, were transcribed verbatim, coded according to issues identified by the participants and analysed thematically. Ethical approval was obtained from the relevant Institutional Review Boards.

Results: Four interlinked themes were identified: physical impact (pain, discomfort, inconvenience); psychosocial wellbeing (embarrassment, loss of self-esteem, frustration, and depression); family consequences (lack of support, self-blame) and concerns about ulcer progression (unpredictable healing, hope).

Conclusions: For the participants, physical impacts, typically pain, were the main concern that 'drives me crazy', suggesting that they were not well managed. They resulted in significant psychosocial issues (including suicidal intent) and other consequences (e.g. relationship issues), of which health professionals might not be aware. Prompt access to holistic multidisciplinary team care and family support in the event of 'emotional crisis' with significant physical impacts could lead to a more positive outlook and improved outcomes. The critical role of cultivating patients' positive psychological resources should never be overlooked or underestimated throughout patients' leg ulcer care journey. Further research on factors specific to Singapore, such as climate and family culture, is recommended to achieve proactive patient centered-care.
\end{abstract}

\section{Keywords}

Leg ulcer, Experience, Primary healthcare, Qualitative study

\section{Introduction}

Leg ulcer is a chronic and debilitating condition affecting $1-3 \%$ of the adult population, with the median duration of 6-9 months and the range of 4 weeks to 72 years [1]. The recurrence rates are up to $70 \%$ within 12 months of healing and a patient may suffer 3-4 recurrent ulcers during their lifetime [2]. Chronic leg ulcer induces both physical and emotional distress, and poses a pervasive and profound effect to patient's Quality of Life (QoL), [3-5] predominantly in the elderly $[6,7]$. It also places a significant economic burden on patients and families, healthcare providers and society. Chronic leg ulcer is usually associated with prolonged ill-health requiring ongoing management over many years or a lifetime. The treatment and management of patients with leg ulcer are mainly carried out in primary healthcare and community care where nurses spend approximately half of their time treating patients with leg ulcers $[3,8]$.

Patients with chronic leg ulcer experience significant unpleasant physical symptoms, including pain, discomfort, copious wound exudate, malodor, altered body image and inconvenience associated with wearing bulky bandages, impaired functional ability and so-

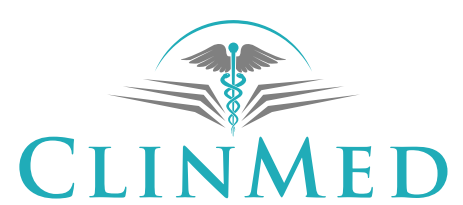

INTERNATIONAL LIBRARY

Citation: Zhu X, Ryan K (2017) 'When Can I be Free from My Miserable Leg?' A Qualitative Study of Patients' Experiences of Chronic Leg Ulceration in Primary Healthcare. Int Arch Nurs Health Care 3:073. doi.org/10.23937/2469-5823/1510073

Received: May 27, 2017: Accepted: July 12, 2017: Published: July 14, 2017

Copyright: (c) 2017 Zhu X, et al. This is an open-access article distributed under the terms of the Creative Commons Attribution License, which permits unrestricted use, distribution, and reproduction in any medium, provided the original author and source are credited. 
cial isolation [4,9-14]. Pain associated with chronic leg ulcer has been reported as chronic [12] and portrayed as 'continuous' and 'unbearable', overwhelmingly dominating the lives of participants [13]. Patients with persistent pain experience sleep disturbance, limited mobility and other negative consequences in relation to poor psychosocial wellbeing such as low mood, insomnia, depression, and suicidal ideation $[6,12,13]$. In addition, the unpredictable nature of exudate and malodor, bulky bandage and altered body image are associated with frustration and social isolation affecting patients' psychological wellbeing [11,13-16] and causing distress, embarrassment, shame and anxiety $[13,14,17]$. Some patients with leg ulcer are at greater risk of depression and exhibit different levels of depressive symptoms $[6,11,14,18,19]$.

The literature postulates that both physical and psychological factors have a negative impact on ulcer healing $[9,19,20]$. This was revealed in a previous study as perceived inconsistency of ulcer treatment and reported problems with regard to follow-up treatment [9]. Physical impacts, such as pain and discomfort, have been identified as the main reasons for non-adherence to leg ulcer treatment $[2,21,22]$. The evidence also suggests a link between depression induced by physical impacts and an inability to comply with treatment [15]. Patients' adherence significantly correlates with wound healing indicating a possible pathway to improved care and outcomes [23]. Therefore, Williams [24] highlighted that adequate understanding of the physical and, psychosocial factors and consideration of the leg ulcer from a patient's perspective could improve patients' concordance with treatment and enhance healing rates possibly resulting in better patient outcomes.

Research evidence indicates that nursing care usually focuses on wound care and the application of bandaging, rather than the patient as a whole or factors that influence Quality of Life (QoL) [3,25]. Jones [26] also claimed that care delivery for people living with leg ulcers still continues to be situated within a biomedical culture, with little attention paid to the psychological and emotional consequences. Hence, healthcare professionals need to fully understand the patients' experience and expectation of living with leg ulceration to deliver quality care $[17,24]$. Although there is a growing body of knowledge about how patients are living with chronic leg ulcers from overseas, in the Singapore context, there is no research in relation to this field. In this light, gaining deep insight into the experiences of living and coping with chronic leg ulcer from this particular group of patients is an area in need of greater exploration. This study, therefore, aimed to explore the experiences of patients with chronic leg ulceration from their perspective in a primary healthcare setting in Singapore. The research question was 'What are the experiences of patients with chronic leg ulceration in primary healthcare?'

\section{Methods}

\section{Study design}

A qualitative exploratory descriptive design employing thematic analysis was used to explore the participants' experience of their 'leg ulcer journey'.

\section{Setting and sample}

A purposive, maximum variation sampling procedure was employed to create depth to the emerging knowledge. The inclusion criteria were adults with ulcer(s) with etiology of venous diseases at lower $\operatorname{limb}(\mathrm{s})$ for longer than 6 weeks, able to give informed consent, and under the care of the local primary healthcare setting for leg ulcer treatment. Patients with arterial ulcers, diabetic foot ulcers, pressure ulcers, and mental disability, were excluded from the study. Eight participants were recruited over six months from the three clinics located in the different regions of the local primary healthcare setting. The same numbers of males and females were recruited to prevent gender bias. The wide age range and ulcer duration were set to ensure that any differences in perspectives according to life stages could be explored. A summary of the characteristics of the participants is presented in Table 1. Semi-structured and audio or video recorded in-depth interviews lasting up to 60 minutes were conducted with participants to record the story of their 'leg ulcer journey'. Interviews included a chronological narrative, a reflective discussion and commentary on the unique aspects of the experience of chronic leg ulcer. Interviews were conducted either in the participant's' home or the Health Education room of the respective clinics at the participant's convenience.

\section{Ethical considerations}

Ethical approval was obtained from the relevant Institutional Review Boards. Before commencing the

Table 1: Summary of participants' characteristics $(n=8)$.

\begin{tabular}{|l|l|l|}
\hline Characteristic & $\begin{array}{l}\text { Sample } \\
\text { representation }\end{array}$ & n (\%) \\
\hline Age in years & & \\
\hline Mean \pm SD (range) & $59 \pm 10$ & \\
\hline Range (minimum-maximum) & $38-86$ & $4(50)$ \\
\hline Gender & & $4(50)$ \\
\hline Male & & \\
\hline Female & & \\
\hline Leg ulcer duration in year & $9 \pm 10$ & \\
\hline Mean \pm SD (range) & $1-50$ & $5(62.5)$ \\
\hline Range & & $2(25)$ \\
\hline Marital status & & $1(12.5)$ \\
\hline Married & & \\
\hline Single & & $6(75)$ \\
\hline Divorced & & $1(12.5)$ \\
\hline Race & & $1(12.5)$ \\
\hline Chinese & & \\
\hline Malay & & \\
\hline Indian & & \\
\hline
\end{tabular}

SD: Standard Deviation. 
study, approval was also obtained from the heads of the three variously located to conduct the study within the primary healthcare organization. Eligible participants were identified and approached by wound nurses in wound dressing rooms. Participants were informed both verbally and in writing about the purpose of the study and invited to participate. A written informed consent was obtained from each willing participant prior to their interview. All participants were reassured that their participation was completely voluntary, and that their refusal to participate in the study would not affect the care they would receive from their clinic. Confidentiality of the interview data and personal identity was guaranteed.

\section{Data collection}

Following the ethical approvals, semi-structured, individual audio or video recorded interviews were held with participants with the help of a flexible interview guide (Table 2). All participants were encouraged to tell their stories by starting with open-ended questions 'Tell me about your experience of having a chronic leg ulcer?' 'What have been your major concerns of your chronic leg ulcer?' Probing questions were used to detail these experiences, such as, 'Would you please give me an example of that?' 'Could you tell me more about that experience?' Subsequently interviews were flexible to allow participants to determine the order in which topics were covered. Time was allowed for responses to be fully probed and to be responsive to issues raised by participants. The topic guide was revised in response to emerging data to allow exploration of new issues with successive participants.

The data collection took place over six months allowing the researcher to develop a deep understanding of participants' experiences of having a chronic leg ulcer. This also enabled the researcher to facilitate and permit the participants to shift their perspectives during the interview process. The interview schedule was used as a topic guide but not in a prescriptive manner, and other questions were asked with relevant tangents explored. Participants were offered different ways of conceptualising an issue. Other questions were added based on new insights gained from the analysis of the previous interviews. These questions focused on deepening the theoretical explanation of the experience of having chronic leg ulcer. Individual interviews lasted up to 60 minutes. Field notes were also taken to enrich and clarify the interview data. Data collection stopped after eight participants had been interviewed because of time constraints and the quality and depth of the data collected was deemed sufficient for analysis.

\section{Data analysis}

The recorded interviews were transcribed verbatim. Expressions, such as exclamations, laughter, and expletives, were included in the text and separated from the verbal text with square brackets, whereas pauses were indicated using dashes [27]. The transcribed data were analysed

Table 2: Interview topic guide.

1. Would you like to tell me something about your experience of having chronic leg ulcer? Do you have further examples of this?

2. What have been your major concerns with your chronic leg ulcer? How do you cope with them?

3. What else can you think of about this topic that I have not asked you about but might be useful to know?

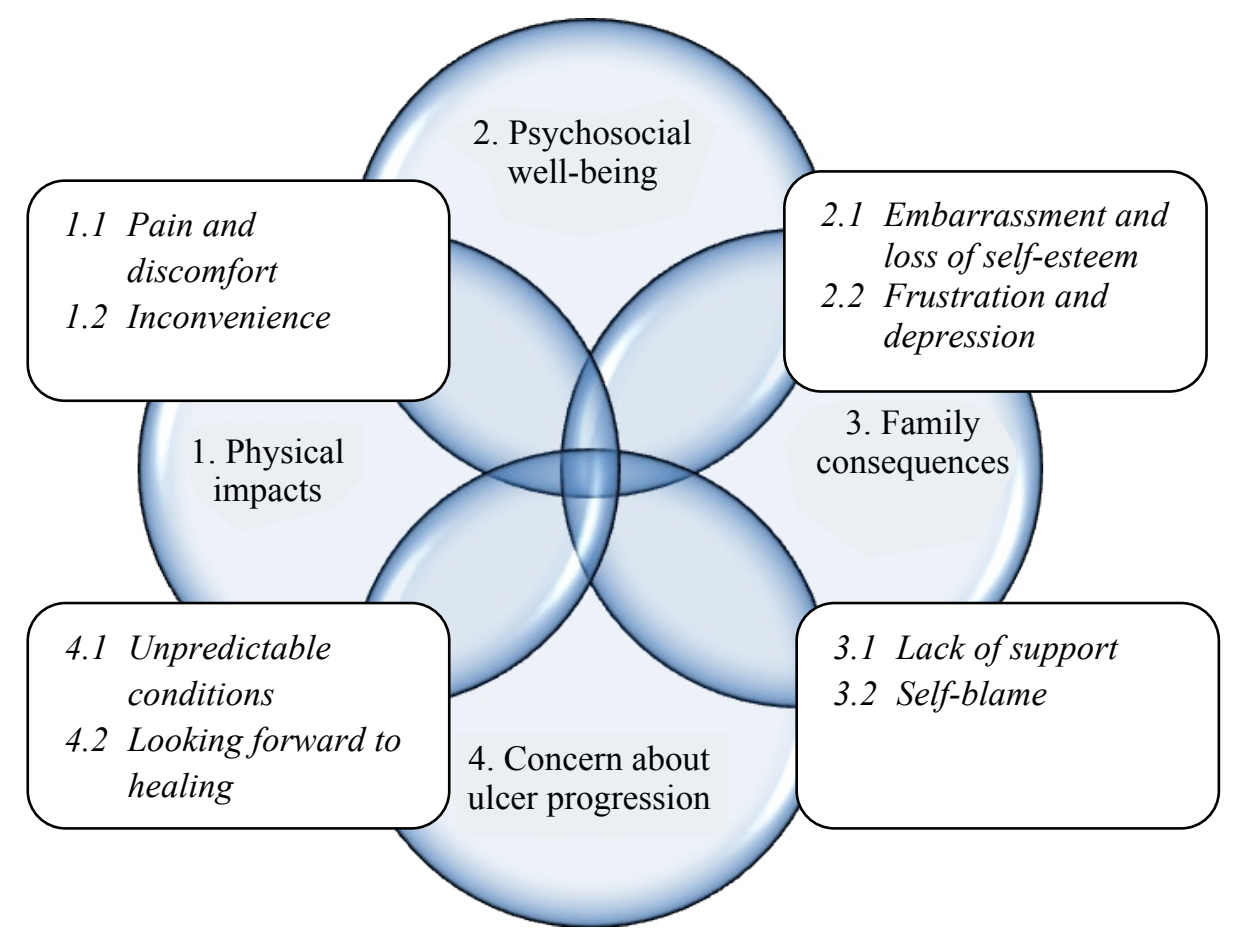

Figure 1: The interlinked themes and subthemes. 
thematically using constant comparison [28], a technique that relies on the interpretation of descriptive data to explicate the significant meanings of the participants' experiences [27]. Data analysis started after the first interview with the researcher reading the transcript several times to ensure complete understanding and familiarity with the data. Any similarities and differences between and within participants' accounts were noted. Word files were created using a personal computer to prepare transcripts and format text for manual coding. Frequently used words, phrases and ideas by participants were identified and organized into codes and categories. The data were coded according to issues identified by the participants themselves, the researcher from her clinical practice and the research literature. The two authors discussed the codes and came to an agreement on them. The coded data were then collapsed into categories of similar codes. The categories were then grouped together into themes and organised into a coherent and internally consistent account. All themes were reviewed and written up as summaries of the topics raised by participants to represent the full range of experiences and to give a realistic impression of what it is like to live with chronic leg ulceration.

\section{Results}

Four main themes emerged from data analysis: physical impacts, psychosocial well-being, family consequences and concern about ulceration progression. The eight emergent subthemes helped to explain how the participants' interviews have addressed the study purpose and answered the research question (Figure 1). Participants' responses related to each theme and subtheme were closely interlinked and overlapped.

\section{Theme 1: Physical impacts}

Subtheme 1.1: Pain and discomfort: Participants described their pain as an overwhelming feature that had a profound effect on their lives. It was mentioned as the first and most dominant experience related to having a chronic leg ulcer.

The pain is like pulling pain, burning pain, and poking pain... It drives me crazy! (Participant 1: female).

Pain is really the 'worst possible symptom'! (Participant 6: female).

Pain really made me tremendously upset! When it comes, I almost think of ending my life early... I have even thought of jumping off a high story (tearing) (Participant 2: female).

The intensity and type of pain described by participants varied according to healthcare interventions. Two participants highlighted that pain worsened after wound dressing, while six participants highlighted that the pain intensity increased at midnight. Leg swelling also exacerbated the pain. All participants experienced increased pain and increased swelling following activities or weight bearing.
The pain always makes me cry after wound dressing is changed. When it (pain) comes, I cannot do anything! I just hope someone can help me 'chop off' my leg and free me from pain! (cried) (Participant 1: female).

The worst part is that I could neither bend my leg nor walk steadily because it is like a huge elephant leg...it is extremely painful! (Participant 2: female).

Participants described the common impact in relation to discomfort caused by heavy exudates and bulky bandages. Discomfort was multi-factorial, and all participants experienced soggy dressing and offensive malodor.

I have been sleeping on the plastic-covered mattress (without bed sheet) for many years! What I need to do is to just wipe away the dirty wound liquid with tissue papers every morning... (Participant 1: female).

The dressing was always wet, my leg has been never dry... it is extremely irritating! (Participant 3: male).

Subtheme 1.2: Inconvenience: Participants explained that chronic leg ulcer has brought inconvenience to their daily life. They described 'inconvenience' as a complex balancing of impaired mobility between having leg ulceration and daily functional activities. They were struggling to perform everyday routine tasks, including difficulties maintaining personal hygiene.

I cannot even give a proper wash to my smelly leg! That's unbelievable but I have been going through this for years! (Participant 8: male).

My roommate did chase me out from the flat because she could not tolerate the smell! (Participant 1: female).

\section{Theme 2: Psychosocial well-being}

Subtheme 2.1: Embarrassment and loss of self-esteem: Most participants denied or intended to deny having chronic leg ulceration with malodor. They displayed various levels of embarrassment which resulted in low self-esteem and low self-confidence. Six participants also repeatedly reported their altered body image and abnormal gait which contributed to their emotional issues and social disconnectedness. Four participants revealed that they always wore long, thick and dark coloured trousers even in the hot weather to hide their bandaged leg in public places.

My colleagues asked me if I am having some strange body odor like rotten fish, I denied ... I don't want them (friends and colleagues) to know I am an ulcer sufferer because I am afraid that they will look down on me. (Participant 6: female).

My friends left me (because of the smell)... I do not think someone wants to be my friend... No point to make friends anymore! (Participant 1: female).

I must wear long pants and long dress with two colors (black and navy blue) to cover my 'crying leg' (wet) 
while most people around me are wearing shorts and nice skirts, isn't it embarrassing? (Participant 1: female).

Subtheme 2.2: Frustration and depression: More than half of the participants spoke of their frustration about having a chronic leg ulcer, and specifically talked about the negative impact on their mood and various levels of disappointment. They described the experience of heavy exudates and offensive malodor as distressing and shameful, especially when 'private' becomes 'public'. Some participants revealed non-compliance to treatment with the attitude of 'I don't care' because of frustration caused by their 'problem leg'.

The lady next to me covered her nose with tissue papers after she looked at my leg. 'My god, she already knew the smell is from my leg!' ..... I have been 'traumatized' many times because of my leg! I was extremely depressed! My heart was aching! (tearing). (Participant 1: female).

I don't want to wear bandages anymore... I don't care about my leg! I am really fed up with my leg! Let it be! (Participant 1: female).

I got no mood to make myself neat and tidy! I am so pissed off!' (Participant 5: male).

\section{Theme 3: Family consequences}

Subtheme 3.1: Lack of support: Typically, this subtheme covers the negative impact of family members on participants. All the married female participants in the interviews were very reluctant and hesitated to voice their 'family stories' in relation to their 'problematic leg' because of concerns about a 'marriage crisis' likely caused by the difficulties with sexual activity or their inability to perform the additional family roles which were expected by their spouse. Two participants even cried and kept a lengthy silence in response to the question of 'family emotional and financial support' although the interviewer probed three times to elicit a wide range of responses.

... (Because of my leg problem), most of the time I am very down! I have no mood to go out (for leisure activities) with him (husband)... Finally he got fed up with me and decided to neither accompanied me for my leg treatment nor paid my medical bill! (sigh) (Participant 6: female).

He was angry with me because I have been always keeping more housework for him... I am really in pain! (Participant 5: female).

We are hardly together (sexual activity) because of (inconvenience caused by) my leg. I am always engaged with my ulcer treatment. He (husband) was very unhappy... We have more arguments than before and quarreled until he wanted a divorce...(tearing) (Participant 6: female).

Subtheme 3.2: Self-blaming: Surprisingly, when mar- ried male participants in this study were asked to identify what was most helpful for coping with their chronic leg ulceration, the most common response focused on support from their spouse and other family members. They also talked about their guilt and regret in relation to the emotional and financial burden that their chronic leg ulcers have placed on their spouse and children. Two participants have almost given up their ulcer treatment as they felt that their ulcer dragged down their sense of self-worth to 'take' and never 'give' for many years.

My (problematic) leg has been creating lots of problems to them (my wife and my children)... I felt regretful and guilty to them ...! (Participant 4: male).

The worst thing about my leg is that whenever I am in pain, my wife suffers with me... I even thought about quitting treatment because it is too troublesome and unfair to my wife (Participant 3: male).

I am useless! ... I cannot imagine my life without my wife... (Participant 4: male).

\section{Theme 4: Concern about ulcer progression}

Subtheme 4.1: Unpredictable conditions: Participants not only highlighted slow healing progress and frequent recurrence as factors affecting their negative feelings and behavior in response to ulceration, they also had a fear of suffering a new ulcer. Some participants voiced their anxiety over falling and causing further injury which made them feel vulnerable and restricted their mobility. They also expressed feelings of fear, sadness and frustration because their ulcer persisted and recurred.

It has not been recovered for so long! It really made me tremendously upset! (Participant 6: female).

It's like a nightmare! It (leg ulcer) always comes back to me! Actually my stress is more on the worries of the ulcer deterioration, recurrence and new ulcer. (Participant 7: male).

Sometimes there is no control over this... Once a small cut (on the leg), a new ulcer again, that's for sure! (Participant 8: male).

Subtheme 4.2: Looking forward to healing: All participants expressed their wish for wound healing. They stressed their desire to regain their quality of life after healing. Though some participants spoke negatively about their ulcer journey, they never stopped hoping for healing and to return to their normal life. This was because they had been going through a prolonged ulcer healing process or had experienced multiple ulcer recurrences before.

It (leg ulcer) just comes back again and again... When can I be free from my miserable leg? (Participant 1).

I hope there is something which can permanently cure it (leg ulcer). (Participant 8: male). 
I am sure it (leg ulcer) will heal one day! (Participant 7: male).

I wish my leg ulcer healed, I can bring my children for outing... (Participant 6: female).

I wish I can wear nice skirt soon! (Participant 2: female).

\section{Discussion}

This study explored the experiences of patients, with chronic leg ulceration in a primary healthcare setting. The findings revealed four interlinked themes (Figure 1) providing deep insights into how patients with chronic leg ulcer had gone through their 'leg ulcer journey'. The study found that patients with chronic leg ulcer suffered higher levels of physical and psychosocial impacts than their 'healthy' peers without leg ulcers. The participants widely highlighted that the physical immobility of living with a chronic leg ulcer had negative impacts on their psychological well-being and resulted in restrictions regarding social functioning, work capacity, housework, recreation, social interaction, as well as causing marital distress and other family unhappiness. Nevertheless, the participants had never stopped expressing their desire for adequate attention for wound healing.

\section{Physical impacts}

Physical impacts were consistently reported in previous studies. Our participants felt that pain restricted their physical activities and was their main concern resulting in significant psychosocial issues and other negative consequences. The participants who experienced severe pain even wanted to release themselves from pain by 'chopping off their leg'. This is consistent with previous studies in which people with chronic leg ulcer suffering severe pain had a desire for limb amputation [12]. The participants also declared that other physical impacts, like heavy exudates, malodour and wet bandages further contributed to feelings of embarrassment and distress. Most of our participants described that leaking exudate and malodour were neither acknowledged nor adequately treated. The findings led us to deduce that the leg ulcer patient group is an 'under-served' group as their pain is under-estimated and under-treated. Proactive symptoms control therefore should be tailored to improving clinical outcomes and returning patients to the greatest level of function both physically and psychosocially. Consistent with previous studies [13-15,29], all participants in this study faced inconvenience and embarrassment due to unsightly bandages contributing to negative body image and limited clothing choices. They had to wear non-preferred long trousers in the hot weather to conceal their bulky or wet bandages, especially on public occasions. This could be associated with the unique Singapore tropical climate; future study is recommended to further explore the relationship between patients' experience and the Singapore climate.

\section{Psychosocial well-being}

The majority of our participants reported that physical stress was the most prominent aspect that eroded their quality of life and made them feel a loss of self-confidence, depression, frustration and despair. They acknowledged that the loss of self-confidence related not only to the activities limited by their chronic leg ulcer but to losing overall confidence in themselves as human beings. Furthermore, they expressed challenges in maintaining a sense of dignity and public denial of having chronic leg ulceration. This finding is in line with a previous study which found that 'when a private condition becomes public', people might experience a sense of loss, low self-esteem and resignation [14]. Consistent with previous studies $[3,11,13,14]$, two participants in this study revealed suicidal intent which was likely caused by persistent pain and the prolonged healing process. They also highlighted their experience of reduced mobility, reduced self-esteem and reduced enjoyment in their lives that contributed to their social isolation and social disconnectedness. This shows that detrimental psychological effects are closely associated with dominance of physical impacts on the individuals. Thus, clinicians' greater awareness and greater emphasis on adopting a multidisciplinary wound care model with a focus on bio-psycho-social care is required to holistically address both significant physical impacts and potential risks of psychological deterioration in this care group.

\section{Family consequences}

Singapore Social Health Project [30] reported that certain aspects of family relationships have shown signs of weakening. However, in this study, three married male participants aged 70 years and over with long-standing chronic leg ulcerations disclosed that their family members (mainly spouses) provided them with strong support both emotionally and financially and aided them in dealing with leg ulcer-related challenges. Some of them have been carrying a sense of 'self-blaming' which further induced a strong sense of guilt about burdening their loved ones for many years. It was also noticed that this group of elderly participants who have been usually independent and dominant occupying a higher status in their family were now unfortunately dependent on their spouse's unconditional support to cope with the disease process. This could be associated with a positive marital relationship or the traditional close-knit nature of families in the Singapore culture. Further study might be needed to determine whether this is so in the local context. On the contrary, though, young married female participants revealed that a poor spousal relationship could be linked to the changes in their mobility and functional ability that originated from their leg pain and restricted activities, including limited participation in joint leisure activities and difficulties with sexual activities. Consistent with the results of a 
systematic review [10], the problem areas our married female participants identified were restrictions in work capacity, recreation, psychological well-being, as well as problems caused by treatment regimens. A recent study by Wellborn and Moceri [31] suggested that support from family members enhances patients' ability to cope with the negative impact of leg ulcer on daily living. A recommendation, therefore, is that since the needs of people living with chronic leg ulceration go beyond their actual wound care, proactive and effective family support for patients, especially married females, is required to facilitate more positive experiences.

\section{Concern about ulcer progression}

The participants in this study illustrated that they were afraid of trauma and injury on the leg. Furthermore, when ulcer healing had occurred, they were often anxious and over conscious of preventing ulcer reoccurrence and developing new ulcers by constantly examining their legs. These findings are in agreement with previous studies that participants were living with continued uncertainty with fear of harm or damage to their ulcer and ulcer recurrence $[5,15,29]$. There is evidence that the majority of patients with chronic leg ulceration had a pessimistic vision of their future and ulcer healing $[10,21]$. Brown showed that patients accepted leg ulcer as a chronic condition and would "never be entirely ulcer-free" [29]. During the interviews, however, our participants revealed that they desperately wanted to be released from their 'problematic leg' permanently. Their perception ("I wish I can bring my children for outing') and the insight ('I can wear my skirt soon') on their wound conditions contributed to the finding of a positive view of 'looking forward to healing'. Apparently, despite negative impacts, our patients' optimistic and positive views towards their healing have informed healthcare professionals that they still have a strong desire for living a normal life. The challenge is now for health professionals to effectively cultivate and appreciate patients' positive psychological resources and develop services that are responsive and proactive to patients' real needs towards greater wound healing rates and better quality of life.

\section{Conclusion}

By exploring the lived experience of patients with chronic leg ulcer, this study adds value to our understanding of a patient's 'leg ulcer journey' in the Singapore context. Apart from exploring a bio-psycho-social model of care, healthcare professionals should be always alert to individual's potential emotional disturbances and psychosocial distress that might lead to relationship issues and marital distress. In the event of 'emotional crisis' (including suicidal intent) accompanied with significant physical impacts (e.g. severe pain), prompt access to holistic multidisciplinary team care and family support could lead to a more positive out- look and improved outcomes. Certainly, the critical role of cultivating positive psychological resources should never be overlooked and underestimated, especially when it is lacking. Further research on Singapore-specific factors, such as climate and family culture in relation to chronic leg ulceration in different age groups, is recommended to achieve proactive patient centered-care.

\section{Conflict of Interest}

The authors have no conflicts of interest to declare.

\section{Acknowledgements}

We thank Ms Chen Yee Chui, Chief Nurse of National Healthcare Group Polyclinics, Singapore, for proofreading the manuscript. We thank the Clinic Heads and the Nurse Managers of the participating clinics, National Healthcare Group Polyclinics, Singapore, for their support to allow access to patients. We truly appreciate all participants for their great support and participation in the study.

\section{References}

1. Briggs M, Closs SJ (2003) The prevalence of leg ulceration: a review of the literature. EWMA J 3.

2. Vowden KR, Vowden $P$ (2006) Preventing venous ulcer recurrence: a review. Int Wound J 3: 11-21.

3. Maddox D (2012) Effects of venous leg ulceration on patients' quality of life. Nurs Stand 26: 42-49.

4. Green J, Jester R, McKinley R, Pooler A (2014) The impact of chronic venous leg ulcers: a systematic review. J Wound Care 23: 601-612.

5. Isaac A, Watson C (2016) How venous leg ulcers affect quality of life. Prim Heal Care 26: 18-23.

6. Ebbeskog B, Ekman SL (2001) Elderly persons' experiences of living with venous leg ulcer: living in a dialectal relationship between freedom and imprisonment. Scand $\mathrm{J}$ Caring Sci 15: 235-243.

7. Hellström A, Nilsson C, Nilsson A, Fagerström C (2016) Leg ulcers in older people: a national study addressing variation in diagnosis, pain and sleep disturbance. BMC Geriatr 16: 25.

8. Morris P, Sander R (2007) Leg ulcers. Nurs Older People 19: 33-37.

9. Persoon A, Heinen MM, van der Vleuten CJ, de Rooij MJ, van de Kerkhof PC, et al. (2004) Leg ulcers: a review of their impact on daily life. J Clin Nurs 13: 341-354.

10. Herber OR, Schnepp W, Rieger MA (2007) A systematic review on the impact of leg ulceration on patients' quality of life. Health Qual Life Outcomes 5: 44.

11. Edwards H, Finlayson K, Skerman H, Alexander K, Miaskowski C, et al. (2014) Identification of symptom clusters in patients with chronic venous leg ulcers. J Pain Symptom Manage 47: 867-875.

12. Taverner T, Closs SJ, Briggs M (2014) The Journey to Chronic Pain: A Grounded Theory of Older Adults' Experiences of Pain Associated with Leg Ulceration. Pain Manag Nurs 15: 186-198.

13. Green J, Jester R, McKinley R, Pooler A (2013) Patient perspectives of their leg ulcer journey. J Wound Care 22: 58 , 60-62, 64-66. 
14. Byrne O, Kelly M (2010) Living with a chronic leg ulcer. J Community Nurs 24: 46.

15. Finlayson K, Edwards H, Courtney M (2010) The impact of psychosocial factors on adherence to compression therapy to prevent recurrence of venous leg ulcers. J Clin Nurs 19 : 1289-1297.

16. Finlayson K, Edwards H, Courtney M (2011) Relationships between preventive activities, psychosocial factors and recurrence of venous leg ulcers: a prospective study. J Adv Nurs 67: 2180-2190.

17. Upton D, Andrews A, Upton P (2017) Venous leg ulcers: What about well-being? J Wound Care 23: 14, 16-17.

18. Brown A (2005) Leg ulcers. Chronic leg ulcers, part 1: do they affect a patient's social life? Br J Nurs 14: 894-898.

19. Moffatt CJ, Franks PJ, Doherty DC, Smithdale R, Steptoe A (2009) Psychological factors in leg ulceration: a case-control study. Br J Dermatol 161: 750-756.

20. Finlayson KJ, Courtney MD, Gibb MA, O'Brien JA, Parker $\mathrm{CN}$, et al. (2014) The effectiveness of a four-layer compression bandage system in comparison to Class 3 compression hosiery on healing and quality of life for patients with venous leg ulcers: a randomized controlled trial. Int Wound J 11: 21-27.

21. Douglas V (2001) Living with a chronic leg ulcer: an insight into patients' experiences and feelings. J Wound Care 10: 355-360.

22. Van Hecke A, Verhaeghe S, Grypdonck M, Beele H, Defloor T (2011) Processes underlying adherence to leg ulcer treatment: a qualitative field study. Int J Nurs Stud 48: 145155.

23. O'Brien J, Finlayson K, Kerr G, Shortridge-Baggett L, Edwards $H$ (2016) Using a theoretical approach to identify factors influencing adherence to an exercise programme for adults with venous leg ulcers. J Health Psychol.

24. Williams AM (2010) Issues affecting concordance with leg ulcer care and quality of life. Nurs Stand 24: 51-52.

25. Heinen MM, Persoon A, van de Kerkhof $P$, Otero $M$, van Achterberg T (2007) Ulcer-related problems and health care needs in patients with venous leg ulceration: a descriptive, cross-sectional study. Int J Nurs Stud 44: 1296-1303.

26. Jones JE (2007) The prevalence and experience of emotional distress in patients with chronic venous ulceration. Liverpool, University of Liverpool.

27. Morse JM, Field PA (1995) Qualitative Research Methods for Health Professionals. ( $2^{\text {nd }}$ edn), Sage Publications, London, England.

28. Pope C, Ziebland S, Mays N (2000) Analysing Qualitative Data. BMJ 320: 114-116.

29. Brown A (2010) Managing chronic venous leg ulcers part 2: time for a new pragmatic approach? J Wound Care 19: 85-94.

30. (2017) Singapore Social Health Project.

31. Wellborn J, Moceri JT (2014) The lived experiences of persons with chronic venous insufficiency and lower extremity ulcers. J Wound Ostomy Continence Nurs 41: 122-126. 